\title{
Frequency of Specific Genes in Different Types of Epilepsy
}

\author{
Neslihan Duzkale ${ }^{1}$ and Rıdvan Akın² \\ ${ }^{1}$ Department of Medical Genetics, Diskapi Yildirim Beyazit Training and Research Hospital, Ankara, Turkey \\ ${ }^{2}$ Department of Pediatric Neurology, Losante Children's and Adult Hospital, Ankara, Turkey
}

\begin{abstract}
Objective: To determine the diagnostic importance of using an exome-based multigene panel in childhood epilepsy.

Study Design: Observational study.

Place and Duration of Study: Department of Medical Genetics, Diskapi Yildirim Beyazid Training and Research Hospital, from January 2017 to May 2020.

Methodology: The phenotype-genotype relationship was investigated in 35 pediatric patients (aged 18 years or younger) with epilepsy, using a large gene panel comprising 464 epilepsy-related genes. The exome-based panel was used to analyse secondary findings.

Results: The diagnostic yield of the targeted multi-gene panel used was 20\% (7/35). The causative genes identified in seven patients (5 boys, 2 girls) were CACNA1E, RELN, PRRT2, TSC1, GABRG2, SCN2A, and SHH. Four of the detected disease-related variants were defined as the novel. Secondary findings in various genes were detected in 19 of the patients. Seven patients with causal genes and the remaining 28 patients were compared in terms of parameters such as gender, mental retardation, developmental retardation, autism, hypotonia, seizure phenotype (only), seizure phenotype (plus), magnetic resonance imaging, degree of kinship of their parents and number of relatives with epilepsy. In addition, patients were evaluated statistically in terms of the same parameters by grouping them according to their gender. There was no statistically significant difference in either study $(p>0.05)$.
\end{abstract}

Conclusion: Genetic testing is an important tool for clinicians in determining the diagnosis, management, and treatment strategies of epilepsy patients.

Key Words: Epilepsy, Diagnostic yield, Exome-based multigene panel, Next-generation sequencing, Seconder findings.

How to cite this article: Duzkale N, Akın R. Frequency of Specific Genes in Different Types of Epilepsy. J Coll Physicians Surg Pak 2021; 31(11):1296-1302.

\section{INTRODUCTION}

Epilepsy is a heterogeneous chronic neurological disease in which spontaneous seizure episodes or convulsions occur as a result of excessive electrical discharge from neurons located mainly in the cerebral cortex. According to the ILAE Commission on Epidemiology, $\geq 2$ seizures that are unprovoked and observed at intervals of $\geq 24$ hours can be considered epilepsy. ${ }^{1}$ The incidence of epilepsy was found to be higher in low/middle-income countries, male gender, youngest and oldest age groups. ${ }^{2}$ Population-based studies have found that 4-5 out of every 1000 children suffer from epilepsy in the period from birth to 15 years of age. The incidence of epilepsy, which is highest in the first year of life in children, is the same as in adults after the age of $10 . .^{2,3}$

Correspondence to: Dr. Neslihan Duzkale, Department of Medical Genetics, Diskapi Yildirim Beyazit Training and Research Hospital, Ankara, Turkey

E-mail: neslihanduzkale@gmail.com

Received: July 27, 2021; Revised: September 18, 2021;

Accepted: September 23, 2021

DOI: https://doi.org/10.29271/jcpsp.2021.11.1296
Although acquired factors such as head injury, tumors, birth trauma and stroke are responsible for approximately one-third of the etiopathogenesis of all epilepsy cases, the remaining part is due to genetics. ${ }^{4}$ In the literature recently, causal variants of many genes involved in the coding ofboth ion and nonionic channels have been reported in association with the epilepsy phenotype. ${ }^{5}$ Therefore, in addition to detailed examination, radiological imaging, and electroencephalogram, genetic tests will be useful in explaining the etiopathogenesis in epilepsy patients. In order to elucidate the highly heterogeneous nature of epilepsy syndromes, next-generation sequencing technology is frequently preferred today in the search for causative genes. Despite its high cost and technical and bioinformatics difficulties, this technique, which allows the analysis of a large number of genes, provides an increase in molecular diagnostic efficiency.

In this study, the genetic etiopathogenesis of the diseases of Turkish patients diagnosed with childhood epilepsy was investigated by the next-generation sequencing (NGS) technique. It was aimed to clarify the relationship between epilepsy phenotype and genotype in the investigated patient group and to determine the diagnostic efficiency of an exome-based multigene panel. 


\section{METHODOLOGY}

This study included 35 patients aged 18 years and younger, with epilepsy phenotype, who were referred to Diskapi Yildirim Beyazit Training and Research Hospital for genetic analysis from January 2017 to May 2020. Demographic and clinical characteristics of the patients, neuroimaging reports, details about patient's and family histories were obtained from patient examination and follow-up files and from the Hospital Electronic database. Family history was evaluated by examining the pedigree analyses of at least 3 generations of the patients. Informed consent was obtained from the parents or legal guardians of all patients participating in the study. This observational study was conducted in accordance with relevant guidelines and regulations, was approved by the independent Ethics Committee of Diskapi Yildirim Beyazit Training and Research Hospital.

Sample preparation, identification of the gene panel and sequencing:

An automated device (Qiagen ${ }^{\circledR}$, USA) was used to extract genomic DNA from the peripheral blood of 35 patients included in the study. DNA sequence analysis was performed with the NGS technique on the Nextseq Platform (Illumina, USA). In this study, the Sophia Clinical Exome Solution ${ }^{\mathrm{TM}}$ (by Sophia Genetics) kit, which allows the analysis of 4490 genes, was used. In the study, analysis was performed with the "Sophia DDM" software, a webbased bioinformatics programme (http://www.sophiagenetics.com/home.html), and 464 genes known to be associated with the epilepsy phenotype were analysed. Guidelines from the "Genomics England PanelApp" database, a public knowledge base, were used to select these genes (http://panelapp.genomicsengland.co.uk/).

The selected genes were "AARS,AARS2,ABAT,ABCA2,ACOX1,A$D A M 22, A D A R, A D A R B 1, A D G R G 1, A D G R V 1, A D R A 2 B, A D S L, A F-$ F3,AGMO,AIMP1,AKT1,AKT3,ALDH5A1,ALDH7A1,ALG1,ALG12,ALG13,ALG14,ALG2,ALG3,ALG6,ALG8,ALG9,AL$P L, A M T, A N K R D 11, A P 3 B 2, A R F G E F 2, A R G 1, A R H G E-$ F9,ARID1B,ARX,ASAH1,ASNS,ASPA,ATN1,ATP1A2,ATP1A3,ATP6AP2,ATP6V0A2,ATP7A,ATRX,BCKDHA,BCKDHB,BCORL1,BCS1L,BOLA3,BRAF,BRAT1,BSCL2,BTD,C12orf57,CACNA1A,CACNA1D,CACNA1E,CACNA1G,CACNA1H,CACNA2D2,CACNB4,CASK,CASR, CBL,CC2D2A,CCDC88C,CDC42BPB,CDKL5,CDON,CEP85L,CHD2,CHRNA2,CHRNA4,CHRNB2,CIC,CLCN2,CLCN4,CLCN6,CLN3,CLN5,CLN6,CLN8,CNKSR2,CNNM2,CNPY3,CNTNAP2,COG4,COG6,COG7,COG8,COL18A1,COL4A1,COL4A2,COQ2,COQ6,COQ9,COX10,COX15,CPA6,CREBBP,CSNK1G1,CST$B, C T S D, C U L 3, C U L 4 B, C Y P 27 A 1, D 2 H G D H, D B T, D C X, D D C, D-$ DX3X,DEAF1,DHCR24,DHCR7,DHDDS,DIAPH1,DISP1,DLL1,DNAJC5,DNAJC6,DNM1,DNM1L,DOCK7,DOLK,DPAGT1,DPM1,DPM2,DPYD,DYNC1H1,DYRK1A,EARS2,EFHC1,EF-

TUD2,EHMT1,EIF2B1, EIF2B2, EIF2B3, EIF2B4,EIF2B5, EIF2S3,EM $X 2, E P M 2 A, E T H E 1, E X O S C 3, E X T 2, F A R S 2, F A S T K D 2, F B X-$

O11,FDFT1,FGF8, FGFR1, FGFR3,FH, FIG4, FKRP, FKTN, FLNA,FOL$R 1, F O X G 1, F O X H 1, F O X R E D 1, F U C A 1, F U T 8, G A B R A 1, G A B R A 2$,GABRB3,GABRD, GABRG2,GAD1,GALC,GALNT2,GAMT,GATM,GBA, GCH1,GCM2, GFAP,GFM1,GLB1,GLDC,GLI2,GLI3,GLRA1,GLR-
B, GLUL, GLYCTK, GM2A,GNAQ,GOSR2,GPHN,GRIN1,GRIN2A,GRIN2B,GRN,GSS,HACE1,HAX1,HCCS,HCFC1,HCN1,HCN2,HEPACAM, HEXA,HEXB, HLCS,HMGCL,HNRNPU,HOXA1, HPRT1,HRAS, HSD17B4,HTRA2,IDH2,IER3IP1,IFIH1,IKBKG,IQSEC2,ISPD,ITPA,KARS, KCNA1,KCND2,KCNJ10,KCNJ11,KCNMA1,KCNQ2,KCNQ3,KCNQ5,KCNT1,KCTD7,KIF1A,KIFIBP, KMT5B,KRAS, LARGE1,LGI1,LIAS, LMNB1,LMNB2,LYST,MADD,MAF,MAGI2,MANBA,MAP2K1,MAP2K2,MAPK10,MBD5,MBOAT7,MECP2,MED12,MED17,MEF2C,MFF,MFSD8,MINPP1,MLC1,MMACHC,MMADHC,MOCS1,MOCS2,MOGS,MPDU1,MTHFR, MTHFS, MTR,NAGA,NARS2,NBEA,NDE1,NDP,NDUFA1,NDUFA10,NDUFA11,NDUFA2,NDUFAF3,NDUFAF4,NDUFAF5,NDUFS1,NDUFS2,NDUFS4,NDUFS6,NDUFS7,NDUFS8,NDUFV1,NEDD4L,NEXMIF,NGLY1,NHLRC1,NODAL,NR4A2,NRAS,NRXN1,NSD1,NSDHL,NTRK2,NUBPL,OCLN,OPHN1,OTX2,PACS1,PAFAH1B1,PAH,PCCA,PCCB,PCDH19,PCDHB4,PCLO,PDHA1,PDHX,PDSS2,PEX1,PEX10,PEX12,PEX13,PEX19,PEX2,PEX3,PEX5, PEX6,PEX7,PHGDH,PIGA,PIGN,PIGO,PIK3CA,PIK3R2,PLA2G6,PLCB1,PMM2,PNKP,PNPO,PNPT1,POLG,POLG2,POMGN-

T1,POMT1,POMT2,PPP3CA,PPT1,PRICKLE1,PRICKLE2, PRMT7,PRODH,PRRT2,PSAP,PSAT1,PSMB8,PSPH,PTCH1,PTEN,PTF1A,PTS,QDPR, RAB18,RAB3GAP1,RAB3GAP2,RALGAPA1,RANBP2,RARS2,RELN,RFT1,RMND1,RNASEH2A, RNASEH2B, RNASEH2C, RNASET2,RNF113A,ROGDI,RO$R A, R P I A, R R M 2 B, R T T N, R U B C N, R Y R 2, S A M H D 1, S C A R B 2, S C-$ $N 1 A, S C N 1 B, S C N 2 A, S C N 2 B, S C N 3 A, S C N 8 A, S C N 9 A, S C 01, S-$ CO2,SDHA,SEPSECS,SERPINI1,SETBP1,SGSH,SHH,SIX3,SLC16A2,SLC25A12,SLC25A19,SLC25A22,SLC2A1,SLC35A1,SLC6A1,SLC6A19,SLC6A5,SLC6A8,SLC9A6,SMAD2,SMARCA2,SMC1A,SMS,SNAP25,SNIP1,SPR,SPTAN1,SRPX2,ST3GAL3,ST3GAL5,STIL,STRADA,STXBP1,SUCLA2,SUCLG1,SUFU,SUOX,SURF1,SYN1,TBC1D24,TBL1XR1,TCF4,TFE3,TGIF1,TMEM70,TPP1,TRAK1,TREX1,TRIP13,TRPM3,TRPM6,TRRAP,TSC1,TSC2,TSEN2,TSEN34,TSEN54,TSFM,TUBA1A,TUBA8,TUBB2B,TUBB3,UBE2A, UBE3A, UBR7, UNC80,VLDLR,WDR45,WDR45B,WDR62,WWOX,ZBTB18,ZDHHC9,ZEB2,ZIC2 and ZNF335".

In the analysis of patient samples included in the study, all exonic regions, 15 base pairs at the exon-intron boundaries and promoter areas of the examined genes were examined and compared with the reference genome (hg19). Variants in these regions were evaluated according to the ACMG (American College of Medical Genetics and Genomics) criteria, and variants classified as pathogenic, likely pathogenic, and of uncertain clinical significance were reported. Variants classified as benign and possibly benign according to ACMG guidelines were ignored in this study. ${ }^{6}$ In silico analysis of variants, DANN, EIGEN, PolyPhen2, Mutation Assessor, Mutation Taster, PROVEAN, GERP, SIFT, CADD, FATHMM, REVEL, LRT, NNSPlice, and Splicesite Finder prediction tools were used.

SPSS (IBM SPSS Statistics 24) programme was used for statistical analysis of the study. $\chi 2$-cross tables were used to compare the two qualitative variables. A resulting $p$-value that was less than or equal to 0.05 was considered statistically significant. 
Table I: Causal variants of patients with epilepsy.

\begin{tabular}{|c|c|c|c|c|c|c|c|c|c|c|c|c|c|c|}
\hline ID/Sex & $\begin{array}{c}\text { Age/Onset } \\
\text { (years) }\end{array}$ & Cons & $\begin{array}{c}\text { Family } \\
\text { History }\end{array}$ & Gene & Cytoband & Nucleotid Change /AA Change & Loc & Zygosity & Func & $\begin{array}{l}\text { Population } \\
\text { Allele Freq } \\
\text { (gnomAD) }\end{array}$ & $\begin{array}{l}\text { ACMG } \\
\text { Scoring }\end{array}$ & dbSNP & Ref & $\begin{array}{c}\text { Gene } \\
\text { Inheritance }\end{array}$ \\
\hline $\mathrm{P} 5 / \mathrm{M}$ & $16 \mathrm{Y} / 11 \mathrm{M}$ & No & 2 & CACNAIE & $1 q 25.3$ & c.702G>A (p.Met234Ile) & E5 & Het & MS & - & LP (PM1,PM2,PP2,PP3) & - & Novel & $A D$ \\
\hline $\mathrm{P} 14 / \mathrm{M}$ & $10 \mathrm{Y} / 8 \mathrm{Y}$ & Yes & 2 & RELN & $7 q 22.1$ & c. $2015 C>T$ (p.Pro672Leu) & E17 & Het & MS & 0.000159 & $\begin{array}{c}\text { LP } \\
(\text { PM1,PM2,PP5,PP3,BP1) }\end{array}$ & rs201044262 & Known & $A D$ \\
\hline $\mathrm{P} 15 / \mathrm{F}$ & $6 Y / 6 M$ & No & 3 & PRRT2 & $16 p 11.2$ & c.649dup (p.Arg217ProfsTer8) & E2 & Het & FS & 0.00374 & P (PVS1,PP5) & rs587778771 & Known & $A D$ \\
\hline $\mathrm{P} 16 / \mathrm{M}$ & $10 Y / 2 Y$ & Yes & 2 & TSC1 & $9 q 34.13$ & c.3277C>T (p.Arg1093Ter) & E23 & Het & NS & - & LP (PVS1,PM2,PP3) & rs1431256375 & Novel & $A D$ \\
\hline $\mathrm{P} 18 / \mathrm{M}$ & $6 Y / 5 Y$ & Yes & 2 & GABRG2 & $5 q 34$ & c.1A>G (p.Met1Val) & E1 & Het & $\mathrm{SL}$ & - & LP (PVS1,PM2,PP3) & rs1581275976 & Novel & $A D$ \\
\hline $\mathrm{P} 23 / \mathrm{M}$ & $11 Y / 4 Y$ & No & 0 & SCN2A & $2 q 24.3$ & c.2944C>T (p.Leu982Phe) & E18 & Het & MS & - & LP (PM1,PM2,PP2,PP3) & - & Novel & $A D$ \\
\hline $\mathrm{P} 25 / \mathrm{F}$ & $12 Y / 3 Y$ & No & 1 & $\mathrm{SHH}$ & $7 q 36.3$ & c. $1246 \mathrm{~A}>\mathrm{G}$ (p.Thr416Ala) & E3 & Het & MS & - & $\begin{array}{c}\text { LP } \\
\text { (PM1,PM2,PP2,PP3,BS3) }\end{array}$ & rs 1412744230 & Known & $A D$ \\
\hline
\end{tabular}

Table II: Variants that do not explain the etiopathogenesis of the epilepsy.

\begin{tabular}{|c|c|c|c|c|c|c|c|c|c|c|c|c|c|c|}
\hline ID/Sex & $\begin{array}{c}\text { Age/Onset } \\
\text { (years) }\end{array}$ & Cons & $\begin{array}{l}\text { Family } \\
\text { History }\end{array}$ & Gene & Cytoband & Nucleotid Change /AA Change & Loc & Zygosity & Func & $\begin{array}{l}\text { Population } \\
\text { Allele Freq } \\
\text { (gnomAD) }\end{array}$ & $\begin{array}{l}\text { ACMG } \\
\text { Scoring }\end{array}$ & dbSNP & Ref & $\begin{array}{c}\text { Gene } \\
\text { Inheritance }\end{array}$ \\
\hline \multirow[t]{2}{*}{ P1/F } & \multirow[t]{2}{*}{$3 Y / 6 \mathrm{M}$} & \multirow[t]{2}{*}{ Yes } & \multirow[t]{2}{*}{0} & SCN1A & $2 q 24.3$ & c.3337G>A (p.Val1113Met) & E19 & Het & MS & - & $\begin{array}{c}\text { VUS } \\
\text { (PM2,PP2,PP3) } \\
\end{array}$ & rs1331336011 & Novel & $A D$ \\
\hline & & & & TSC1 & $9 q 34.13$ & c.1336G>A (p.Glu446Lys) & E14 & Het & MS & - & VUS (PM2,PP3) & - & Novel & $A D$ \\
\hline P18/M & $6 Y / 5 Y$ & Yes & 2 & CACNAIH & $16 \mathrm{p} 13.3$ & c.6557_6559del (p.Lys2186del) & E35 & Het & IF & 0.0000521 & $\begin{array}{c}\text { VUS } \\
\text { (PM2,PM4,BS2) }\end{array}$ & rs766893201 & Novel & $A D$ \\
\hline P21/F & $17 Y / 8 Y$ & No & 0 & RELN & $7 q 22.1$ & c.5330T>C (p.lle1777Thr) & E35 & Het & MS & - & VUS (PM2,BP1) & - & Novel & $A D$ \\
\hline \multirow[t]{2}{*}{$\mathbf{P 3 1} / \mathbf{F}$} & \multirow[t]{2}{*}{$14 \mathrm{Y} / 3 \mathrm{M}$} & \multirow[t]{2}{*}{ Yes } & \multirow[t]{2}{*}{1} & GABRD & $1 p 36.33$ & c.60_61insCAGGTG (p.Arg20_Gly21insGInVal) & E1 & Het & IF & - & $\begin{array}{c}\text { VUS } \\
\text { (PM2,PM4) }\end{array}$ & - & Novel & $A D$ \\
\hline & & & & GRIN2A & $16 \mathrm{p} 13.2$ & c.998C >A (p.Thr333Asn) & E3 & Het & MS & 0.000004 & VUS (PM2,PP2) & rs370306281 & Novel & $A D$ \\
\hline
\end{tabular}

TSC1(NM_000368.5), CACNA1H(NM_021098.3), GABRD(NM_000815.5), GRIN2A(NM_001134407.3).

Table III: Secondary findings (recommended to be reported in the ACMG guidelines).

\begin{tabular}{|c|c|c|c|c|c|c|c|c|c|c|c|c|c|c|c|c|}
\hline ID/Sex & $\begin{array}{c}\text { Age/Onset } \\
\text { (years) }\end{array}$ & Cons & $\begin{array}{l}\text { Family } \\
\text { History }\end{array}$ & Gene & Cytoband & Nucleotid Change /AA Change & Loc & Zygosity & Func & \begin{tabular}{|c|} 
Population \\
Allele Freq \\
(gnomAD)
\end{tabular} & $\begin{array}{l}\text { ACMG } \\
\text { Scoring }\end{array}$ & dbSNP & Ref & $\begin{array}{c}\text { Gene } \\
\text { Inheritance }\end{array}$ & Phenotype & омІм \\
\hline $\mathbf{P} \mathbf{1 / F}$ & $3 Y / 6 M$ & Yes & 0 & RYR1 & $19 q 13.2$ & c.8330A>G (p.Tyr2777Cys) & E54 & Het & MS & 0.0000401 & $\begin{array}{c}\mathrm{LP} \\
\text { (PM1,PM2,PP2,PP3) }\end{array}$ & rs769276412 & Novel & $A D$ & $\begin{array}{c}\text { Malignant } \\
\text { hyperthermia } \\
\text { susceptibility } 1\end{array}$ & 145600 \\
\hline $\mathrm{P} 7 / \mathrm{M}$ & $9 Y / 9,5 \mathrm{M}$ & No & 1 & MYH7 & $14 q 11.2$ & $c .2140 C>A(p . L e u 714 \| l)$ & E19 & Het & MS & - & $\begin{array}{l}\text { LP (PM1,PM2, } \\
\text { PP2,PP3) }\end{array}$ & - & Novel & $A D, A R$ & $\begin{array}{c}\text { Cardiomyopathy, } \\
\text { Myopathy }\end{array}$ & 160760 \\
\hline P19/M & $13 Y / 8 Y$ & No & 0 & $A P C$ & $5 q 22.2$ & c.3920T>A (p.Ile1307Lys) & E16 & Het & MS & 0.00201 & LP (PM2,PP5) & rs1801155 & Known & $A D$ & $\begin{array}{l}\text { Adenomatous } \\
\text { polyposis coli, }\end{array}$ & 175100 \\
\hline $\mathbf{P} 21 / \mathrm{F}$ & $17 Y / 8 Y$ & No & 0 & $A P C$ & $5 q 22.2$ & c.3920T>A (p.Ile1307Lys) & E16 & Het & MS & 0.00201 & LP (PM2,PP5) & rs1801155 & Known & $A D$ & $\begin{array}{l}\text { Adenomatous } \\
\text { polyposis coli, }\end{array}$ & 175100 \\
\hline P32/M & $14 Y / 2 Y$ & Yes & 0 & DSG2 & $18 q 12.1$ & $c .378+1 G>T$ & 14 & Het & SE & - & LP (PVS1,PM2,PP3) & - & Novel & $A D$ & $\begin{array}{c}\text { Arrhythmogenic } \\
\text { right ventricular } \\
\text { dysplasia } 10 \\
\text { Cardiomyopathy, } \\
\text { dilated, } 1 \mathrm{BB}\end{array}$ & 125671 \\
\hline P33/M & $10 Y / 6 M$ & No & 1 & RYRI & $19 q 13.2$ & c.11813G>A (p.Gly3938Asp) & E86 & Het & MS & - & $\begin{array}{c}\text { LP } \\
(\mathrm{PM} 1, \mathrm{PM} 2, \mathrm{PP} 2, \mathrm{PP} 3)\end{array}$ & - & Known & $A D$ & $\begin{array}{c}\text { Malignant } \\
\text { hyperthermia } \\
\text { susceptibility } 1\end{array}$ & 145600 \\
\hline
\end{tabular}

\section{RESULTS}

A total of 35 children, 17 boys (48.6\%) and 18 girls ( $51.4 \%)$, were included in this study. The mean age of the patients included in the study was $11.45 \pm 4.05$ years and the mean age at which epilepsy phenotypes were first observed was $4.72 \pm 4.23$ years. Of the patients, $14(40 \%)$ had growth retardation and the remaining $21(60 \%)$ were within the normal growth percentiles. Mental retardation was diagnosed in 21 patients (60\%), of which 6 (28.5\%) were severe, and 5 of them had autism findings. There were clinical findings of hypotonia in 5 patients with moderate and severe mental retardation. Five (14.3\%) parents were first-degree cousins, $3(8.6 \%)$ were second-degree cousins, and 5 (14.3\%) were distant relatives and/or the same villager. There was no consanguinity between the parents of the remaining 22 (62.9\%) patients. In the pedigree analyzes of the patients, it was determined that $18(51.4 \%)$ patients had at least 2 relatives with epilepsy. Of the patients, 5 (14.3\%) had more than 3 relatives with epilepsy, and the remaining $12(34.3 \%)$ patients had no relatives with epilepsy. While epilepsy was the only clinical finding in 11 (31.4\%) of the patients, behavioural and developmental phenotypes (developmental delay, speech/language delay, hyperactivity or autism, etc.) were also observed in 24 (68.6\%) patients.

As a result of genetic analysis, causal variants that the authors think to play a role in genetic etiopathogenesis were detected in 7 (20\%) of the patients (Table I).

Three of the patients (8.6\%) had VUS variants in genes, associated with the epilepsy phenotype; and the remaining 25 (71.4\%) patients were considered normal for these genes (Table II). 
Table IV: Secondary findings (genes not mentioned in ACMG guidelines).

\begin{tabular}{|c|c|c|c|c|c|c|c|c|c|c|c|c|c|c|c|c|}
\hline ID/Sex & $\begin{array}{c}\text { Age/Onset } \\
\text { (years) }\end{array}$ & Cons & $\begin{array}{l}\text { Family } \\
\text { History }\end{array}$ & Gene & Cytoband & Nucleotid Change /AA Change & Loc & Zygosity & Func & $\begin{array}{l}\text { Population } \\
\text { Allele Freq } \\
\text { (gnomAD) }\end{array}$ & $\begin{array}{l}\text { ACMG } \\
\text { Scoring }\end{array}$ & dbSNP & Ref & $\begin{array}{c}\text { Gene } \\
\text { Inheritance }\end{array}$ & Phenotype & OMIM \\
\hline \multirow{3}{*}{$\mathrm{P} 1 / \mathrm{F}$} & \multirow{3}{*}{$3 \mathrm{Y} / 6 \mathrm{M}$} & \multirow{3}{*}{ Yes } & \multirow{3}{*}{0} & RP1L1 & $8 p 23.1$ & c.5959C>T (p.Gln1987Ter) & E4 & Het & NS & 0.00163 & $\mathrm{P}(\mathrm{PVS1} 1, \mathrm{PM} 2, \mathrm{PP} 5)$ & rs200846354 & Known & $A D$ & Occult macular dystrophy & 613587 \\
\hline & & & & SLC34A1 & $5 q 35.3$ & c.272_292del (p.Val91_Ala97del) & E4 & Het & IF & 0.0168 & LP (PM2, PM4,PP5) & rs876661296 & Known & $A D$ & $\begin{array}{c}\text { Nephrolithiasis/osteoporosis, } \\
\text { hypophosphatemic, } 1\end{array}$ & 612286 \\
\hline & & & & CAPN3 & $15 q 15.1$ & c.1621C>T (p.Arg541Trp) & E13 & Het & MS & 0.0000358 & $\mathrm{P}(\mathrm{PM} 2, \mathrm{PM} 5, \mathrm{PP} 3)$ & rs142004418 & Known & $A D$ & $\begin{array}{c}\text { Muscular dystrophy, limb- } \\
\text { girdle, autosomal dominant } \\
4\end{array}$ & 618129 \\
\hline $\mathrm{P} 2$ & $18 \mathrm{Y} / 11 \mathrm{Y}$ & Yes & Yes & VWF & $12 \mathrm{p} 13.31$ & c.2561G>A (p.Arg854GIn) & E20 & Het & MS & 0.0034 & $\mathrm{P}(\mathrm{PM} 2, \mathrm{PM} 5, \mathrm{PP} 2, \mathrm{PP} 5)$ & rs41276738 & Known & $A D, A R$ & von Willebrand disease & 613160 \\
\hline $\mathrm{P} 4 / \mathrm{F}$ & $9 \mathrm{Y} / 8 \mathrm{Y}$ & No & 2 & NOTCH3 & $19 \mathrm{p} 13.12$ & c.446G>T (p.Gly149Val) & E4 & Het & MS & - & LP (PM1,PM2,PM5, PP2,PP3) & rs1188389375 & Novel & $A D$ & $\begin{array}{c}\text { Myofibromatosis, infantile 2, } \\
\text { Cerebral arteriopathy with } \\
\text { subcortical infarcts and } \\
\text { leukoencephalopathy 1, } \\
\text { Lateral meningocele } \\
\text { syndrome }\end{array}$ & 600276 \\
\hline \multirow{2}{*}{$\mathrm{P} 9 / \mathrm{F}$} & \multirow{2}{*}{$14 \mathrm{Y} / \mathrm{NA}$} & \multirow{2}{*}{ No } & \multirow{2}{*}{9} & MEFV & $16 \mathrm{p} 13.3$ & c.2080A>G (p.Met694Val) & E10 & Het & MS & 0.000282 & P(PP5,PM1,PM2,PM5,PP2,BP4) & rs61752717 & Known & $A D, A R$ & Familial Mediterranean fever & 608107 \\
\hline & & & & RNASEL & $1 q 25.3$ & c.793G $>$ T (p.Glu265Ter) & E2 & Het & NS & 0.00362 & P(PVS1,PM2,PP5) & rs74315364 & Known & $A D$ & Prostate cancer 1 & 601518 \\
\hline P12/M & $10 Y / 2 Y$ & Yes & 0 & $A D S L$ & $22 q 13.1$ & c.1277G>A (p.Arg426His) & E12 & Hom & MS & 0.000199 & P(PM1,PM5,PP2,PP3,PP5) & rs119450941 & Known & $A R$ & Adenylosuccinase deficiency & 103050 \\
\hline \multirow[t]{2}{*}{ P14/M } & \multirow[t]{2}{*}{$10 \mathrm{Y} / 8 \mathrm{Y}$} & \multirow[t]{2}{*}{ Yes } & \multirow[t]{2}{*}{2} & ATP1A2 & $1 q 23.2$ & c.1276T>C (p.Cys426Arg) & E10 & Het & MS & - & VUS(PM2,PP2,PP3) & - & Novel & $A D$ & $\begin{array}{c}\text { Alternating hemiplegia of } \\
\text { childhood } 1 \text {, Migraine, } \\
\text { familial }\end{array}$ & 182340 \\
\hline & & & & HPS1 & $10 q 24.2$ & c. $325 \mathrm{C}>\mathrm{T}$ (p.Gln109Ter) & E5 & Hom & NS & 0.000012 & LP (PVS1,PM2) & rs772192145 & Novel & AR & $\begin{array}{l}\text { Hermansky-Pudlak } \\
\text { syndrome } 1\end{array}$ & 203300 \\
\hline \multirow[b]{2}{*}{$\mathrm{P} 17 / \mathrm{M}$} & \multirow[b]{2}{*}{$18 Y / 1 Y$} & \multirow[b]{2}{*}{ Yes } & \multirow[b]{2}{*}{0} & MEFV & $16 \mathrm{p} 13.3$ & c.2080A>G (p.Met694Val) & E10 & Het & MS & 0.000282 & $\mathrm{P}(\mathrm{PP} 5, \mathrm{PM} 1, \mathrm{PM} 2, \mathrm{PM} 5, \mathrm{PP} 2, \mathrm{BP} 4)$ & rs61752717 & Known & $A D, A R$ & Familial Mediterranean fever & 608107 \\
\hline & & & & HNMT & $2 q 22.1$ & c.623T>C (p.Leu208Pro) & E6 & Het & MS & 0.00000398 & LP (PM2,PS3,PP5) & rs745756308 & Known & $A D, A R$ & $\begin{array}{l}\text { Asthma, susceptibility to, } \\
\text { Mental retardation, } \\
\text { autosomal recessive } 51\end{array}$ & 605238 \\
\hline $\mathrm{P} 22 / \mathrm{F}$ & $14 \mathrm{Y} / 7 \mathrm{M}$ & Yes & 2 & $\mathrm{RDH} 12$ & $14 q 24.1$ & $c .464 C>T(p . T h r 155 \| l e)$ & E7 & Het & MS & 0.0000199 & LP (PM2,PP2,PP3,PP5) & rs121434337 & Known & $A D, A R$ & $\begin{array}{c}\text { Leber congenital amaurosis } \\
13\end{array}$ & 612712 \\
\hline P23/M & $11 Y / 4 Y$ & No & 0 & MEFV & $16 \mathrm{p} 13.3$ & c.2040G>C (p.Met680Ile) & E10 & Het & MS & 0.000103 & P(PS1,PM5,PM1,PM2,PP5) & rs28940580 & Known & $A D, A R$ & Familial Mediterranean fever & r 608107 \\
\hline $\mathrm{P} 26 / \mathrm{M}$ & $8 Y / 2.5 Y$ & No & 1 & PSEN2 & $1 q 42.13$ & c.1289C>T (p.Thr430Met) & E13 & Het & MS & 0.0000359 & LP (PM1,PP2,PP3,PP5) & rs63750666 & Known & $A D$ & $\begin{array}{c}\text { Alzheimer disease- } 4 \\
\text { Cardiomyopathy, dilated, } 1 \mathrm{~V}\end{array}$ & 600759 \\
\hline \multirow{2}{*}{$\mathrm{P} 29 / \mathrm{F}$} & \multirow{2}{*}{$9 \mathrm{Y} / 6 \mathrm{M}$} & \multirow{2}{*}{ No } & & MEFV & $16 \mathrm{p} 13.3$ & c.2230G>T (p.Ala744Ser) & E10 & Het & MS & 0.00184 & LP (PM1,PM2, PP2,PP5) & rs61732874 & Known & $A D, A R$ & Familial Mediterranean fever & 608107 \\
\hline & & & & MEFV & $16 \mathrm{p} 13.3$ & c.2080A>G (p.Met694Val) & E10 & Het & MS & 0.000282 & P(PP5,PM1,PM2,PM5, PP2,BP4) & rs61752717 & Known & $A D, A R$ & Familial Mediterranean fever & r 608107 \\
\hline P30/M & $18 Y / 13 Y$ & No & 0 & MEFV & $16 \mathrm{p} 13.3$ & c.2080A>G (p.Met694Val) & E10 & Het & MS & 0.000282 & P(PP5,PM1,PM2,PM5,PP2,BP4) & rs61752717 & Known & $A D, A R$ & Familial Mediterranean fever & 608107 \\
\hline P34/M & $13 Y / 6 Y$ & Yes & 1 & OPTN & $10 \mathrm{p} 13$ & c. $1242+1 \mathrm{G}>\mathrm{A}$ & 110 & Het & SE & 0.000004 & LP (PVS1,PM2) & rs1206478143 & Novel & $A D$ & \begin{tabular}{|c|} 
Glaucoma 1, open angle, E, \\
Amyotrophic lateral \\
sclerosis 12 with or without \\
frontotemporal dementia
\end{tabular} & 602432 \\
\hline
\end{tabular}

In this study, the causative genes of the patients whose genetic etiopathogenesis was disclosed were determined as CACNA1E, RELN, PRRT2, TSC1, GABRG2, SCN2A and SHH. In 6 of 7 patients with genetic etiopathogenesis of epilepsy, additional developmental and behavioral disorders such as mental retardation, growth retardation and hyperactivity were observed in addition to seizures.

It is recommended in the ACMG guidelines to report the causal variants of 73 genes obtained in clinical exome and genome sequencing as secondary findings. The 73 genes reported in this guideline can highly penetrate and, they have conditions for effective intervention and treatment. ${ }^{7}$

The ACMG seconder finding genes are ACVRL1,RB1,RET,RYR1, RYR2, HFE, PTEN, PRKAG2,PCSK9,BRCA1,BRCA2,STK11,ATP7B,TMEM43,SCN5A,MEN1, DSP,KCNH2,MYBPC3,GAA,TPM1,TSC2,SDHC, TSC1, SDHD, HNF1A, SDHB, TGFBR1, TGFBR2, TRDN, ACTA2, MSH6, BTD, MSH2, SDHAF2,KCNQ1, MYL2,TNNT2, MYL3,CASQ2, PKP2,DSG2,TP53,DSC2, ENG, MYH7, MAX, TTN, TMEM127, LMNA, PMS2, MYH11, TNNI3, CACNA1S, FLNC, VHL,APOB, LDLR, MUTYH, SMAD4, SMAD3, MLH1,PALB2, RPE65,COL3A1,ACTC1,APC,WT1,NF2,GLA,BMPR1A,FBN1 and OTC.

In six of the patients in this study, causal variants were detected in the RYR1, MYH7, APC, and DSG2 genes, which are on the ACMG secondary findings genes list (Table III).

Causal variants of some genes not included in this list were detected in 13 patients in our study in addition to secondary findings (Table IV).
With this genetic analysis, the authors divided seven patients with causal gene variants and the remaining 28 patients into 2 groups and compared them statistically in terms of some variables. There was no statistically significant relationship between the groups in terms of parameters such as gender $(p=0.22)$, mental retardation $(p=0.43)$, developmental delay $(p=0.09)$, autism $(p=0.55)$, hypotonia ( $p=0.55)$, seizure phenotype (only) $(p=0.39$ ), seizure phenotype (plus) $(p=0.39), M R I(p=0.24)$, degree of parental consanguinity $(p=0.41)$, and the number of relatives with epilepsy $(p=0.185)$.

The authors also grouped the patients according to their gender and compared the groups in terms of parameters such as mental retardation ( $p=0.31)$, developmental delay $(p=0.17)$, autism $(p=0.65)$, hypotonia $(p=1.00)$, seizure phenotype (only) $(p=0.14)$, seizure phenotype (plus) ( $p$ $=0.14)$, MRI $(p=0.36)$, degree of parental consanguinity ( $p$ $=0.25)$, and the number of relatives $(p=0.08)$. Statistically, no significant relationship was found between gender and these variables.

\section{DISCUSSION}

Epilepsy is a multifactorial heterogeneous group of diseases in which many genetic, individual, and environmental causes play a role in its etiopathogenesis. Thanks to recent genetic studies, significant progress has been made in the discovery of the causative genes behind this common neurological condition. Data obtained from studies to understand the genetics of epilepsy may not only benefit patients suffering 
from this condition in diagnosis, but may also offer new possibilities in terms of treatment. Studies in the literature have shown that the diagnostic yield of many epilepsy gene panels varies between $10 \%$ and $50 \%$, depending on the selected patient group and the genes covered by the panel. Although the first studies were carried out in patient groups with epileptic encephalopathy, there are genetic studies of many epilepsy cases due to the fact that genetic analyzes have become more accessible today. ${ }^{8-12}$

A recent meta-analysis compared the diagnostic yields and cost-effectiveness of chromosomal microarray (CMA), epilepsy panel with deletion/duplication testing (EP), and whole exome (WES) tests. Diagnostic yield rates were determined as WES, EP, and CMA, from high to low. $(0.45,0.23$, and 0.08 respectively). In terms of cost-effectiveness, WES and EP were close to each other, and the cost-effectiveness of CMA was found to be less than the others. Therefore, CMA was not recommended as the first-line test in epilepsy. ${ }^{13}$

The diagnostic yield of the gene panel used in this study was found to be $20 \%$ (7/35). One of the patients in the study had a novel likely pathogenic variant in the CACNA1E gene. Expressed in both the central nervous system and the endocrine system, the CACNA1E (MIM: 601013) gene is involved in encoding a critical alpha-1E subunit of a calcium channel associated with rapid synaptic transmission in the brain. The relationship between the CACNAIE gene and epilepsy was defined for the first time in the literature in a study conducted on 30 individuals diagnosed with developmental and epileptic encephalopathy. In this autosomal dominant inherited disease, the developmental disorder is accompanied by refractory seizures, hypotonia, contractures, hyperkinetic movements and often macrocephaly. ${ }^{14}$ The patients in this study had growth retardation, seizures that started in the first year of life, and mild mental retardation. It is known that the use of the topiramate has positive results in the treatment of patients with CACNAIE as the causative gene in the etiopathogenesis of their epilepsy. Detection of the causal variant by genetic analysis in the patients made a significant contribution to the decision of treatment options.

In another patient in the study, the causative gene RELN was identified. The RELN gene (MIM:616436) is responsible for the autosomal dominant lateral temporal epilepsy phenotype. In this type of epilepsy with variable penetrance and benign prognosis, focal seizures with auditory aura are observed. In addition, findings such as vertigo, headache, and epigastric discomfort may be observed. Patients respond well to drug therapy. ${ }^{15}$

In another patient, PRRT2 was identified as the causative gene. The PRTT2 gene (MIM:614386) is responsible for the benign familial infantile epilepsy clinic, which is inherited as an autosomal dominant, usually occurs in the first year of life, has a good drug response and does not leave sequelae.
This gene, which is most frequently expressed in the cerebellum of the brain, is thought to play a role in neurodevelopment. $^{16}$

A disease-associated variant in the TSC1 gene was detected in one of the patients in the study. Tuberous sclerosis complex 1 (MIM:605284) is a heterogeneous autosomal dominantly inherited disease in which mental retardation, epilepsy and various organ anomalies are observed and causal variants of TSC1 and TSC2 genes are responsible. In at least $80 \%$ of TSC-associated epilepsies, resistance to multiple antiepileptic drugs is observed, and patients often have severe neurodevelopmental disorders. ${ }^{17}$ This patient had severe mental retardation and growth retardation.

In another patient, a causative variant was found in the GABRG2 gene. The GABRG2 gene (MIM:137164) encodes a member of the GABA-A receptor gene family, which is the site of action for a number of pharmacological agents such as barbiturates, benzodiazepines, and ethanol. These receptors are involved in the modulation of the excitability of the neurotransmitter network in the brain. Dysfunctions of the $\mathrm{y} 2$ subunit encoded by GABRG2 cause more than half of the epilepsies caused by the GABA-A receptor family. Causal variants of GABRG2 cause a variable phenotype, from epilepsies with a fairly good prognosis to severe epilepsies. ${ }^{18}$ This patient in the study had no clinical findings other than epilepsy and headache.

Another causative gene identified in this study was SCN2A. The SCN2A gene (MIM:182390) encodes NaV1.2, a voltage-gated sodium channel that initiates and conducts action potentials. In the causal variants of this gene, a wide spectrum of clinical manifestations can be observed, ranging from benign familial infantile seizures to severe phenotypes such as developmental and epileptic encephalopathies. ${ }^{19}$ The phenotypic findings of the patients were compatible with the clinic of developmental and epileptic encephalopathy 11.

As a result of the analysis, the causative $\mathrm{SHH}$ gene was detected in a patient with growth retardation and mild mental retardation. The SHH gene (MIM:600725) encodes an important member of the hedgehog family, which plays a critical role in the organization and morphology of embryonic development during the intrauterine period. The protein expressed from the $\mathrm{SHH}$ gene is not only important for neuronal development of the central nervous system during the embryonic stage. In adulthood, it has a role in neural tissue hemostasis, control of proliferation of neurons and aging. ${ }^{20}$ Studies in the literature have proven that $\mathrm{SHH}$ modulates neuronal electrical activity in the adult brain and is also an important regulator in the formation of glutamatergic and GABAergic terminals of neurons in the hippocampal region. ${ }^{21,22}$ Studies have also shown that SHH protein, which is released very rapidly in epileptic conditions, plays a role in the regulation of extracellular glutamate levels and thus may affect the development of epilepsy. ${ }^{23}$ 
Test results that provide information about disease-related variants detected in genes associated with a different phenotype, unrelated to the genetic analysis indication of the patients, are called secondary findings. The large gene panel used in this study includes secondary finding genes recommended for reporting in ACMG guidelines. The secondary findings report will ensure that the patient is included in observation, screening and primary symptom prevention programs before the relevant clinical findings emerge. If the patient is symptomatic in terms of secondary findings, will have the chance of the intensive screening correct diagnosis and appropriate treatment. Predictive genetic testing is not recommended in children and adolescents, especially for adult-onset diseases. However, with the secondary findings report, parents and relatives may have the opportunity to incidentally discover a life-threatening risk factor.

Although the sample size of the study was relatively small, this is the first study in investigating a large number of genes in our population. The inability of NGS analysis to detect large deletions and duplications and structural chromosomal aberrations may have contributed to the failure to detect the causative gene in the remaining patients. Although the panel used covers a large number of genes, WES or whole-genome sequencing may be beneficial in patients in whom causative genes cannot be detected.

\section{CONCLUSION}

The diagnostic yield of NGS, using a targeted multigene panel in pediatric patients with epilepsy, was found to be $20 \%(7 / 35)$. The causative genes identified in the study were CACNA1E, RELN, PRRT2, TSC1, GABRG2, SCN2A and SHH. In epilepsy, which is a heterogeneous group of diseases. Diagnosis of patients with NGS does not only prevent some other unnecessary and invasive diagnostic procedures, it is also a valuable tool for clinicians in reaching optimal treatment and clinical management of these patients.

\section{ETHICAL APPROVAL:}

The present study involved human participants, and it was conducted considering ethical responsibilities according to the World Medical Association and the Declaration of Helsinki. The independent Ethics Committee of the Diskapi Yildirim Beyazit Training and Research Hospital approved this study.

\section{PATIENTS' CONSENT:}

Written informed consents were obtained from all patients who participated in this study.

\section{CONFLICT OF INTEREST:}

The authors declared no conflict of interest.

\section{AUTHORS' CONTRIBUTION:}

ND, RA, Contributed to the understanding and design, analysed and interpreted data, prepared the article, critically reviewed, and approved the final version to be published.
All authors agreed to be accountable for all aspects of work ensuring integrity and accuracy.

\section{REFERENCES}

1. Thurman DJ, Beghi E, Begley CE, Berg AT, Buchhalter JR, Ding $D$, et al. Standards for epidemiologic studies and surveillance of epilepsy. Epilepsia 2011; 52 Suppl 7:2-26. doi:10.1111/j.1528-1167.2011.03121.x

2. Beghi E. The Epidemiology of Epilepsy. Neuroepidemiol 2020; 54(2):185-191. doi:10.1159/000503831.

3. Prasad AN, Sang X, Corbett BA, Burneo JG. Prevalence of childhood epilepsy in Canada. Can J Neurol Sci 2011; 38(5):719-722. doi:10.1017/s0317167100054081.

4. Hildebrand MS, Dahl HH, Damiano JA, Smith RJ, Scheffer IE, Berkovic SF. Recent advances in the molecular genetics of epilepsy. J Med Genet 2013; 50(5):271-9. doi:10.1136/jmedgenet-2012-101448.

5. Symonds JD, Zuberi SM, Johnson MR. Advances in epilepsy gene discovery and implications for epilepsy diagnosis and treatment. Curr Opin Neurol 2017; 30(2):193-199. doi:10. 1097/WC0.00000000000000433.

6. Richards S, Aziz N, Bale S, Bick D, Das S, Gastier-Foster J, et al. Standards and guidelines for the interpretation of sequence variants: A joint consensus recommendation of the American College of Medical Genetics and Genomics and the association for molecular pathology. Genet Med 2015; 17(5):405-24. doi:10.1038/gim.2015.30.

7. Miller DT, Lee K, Chung WK, Gordon AS, Herman GE, Klein TE, et al. ACMG SF v3.0 list for reporting of secondary findings in clinical exome and genome sequencing: A policy statement of the American College of Medical Genetics and Genomics (ACMG) [published online ahead of print, 2021 May 20]. Genet Med 2021; 10.1038/s41436-021-01172-3. doi:10.1038/s41436-021-01172-3.

8. Rim JH, Kim SH, Hwang IS, Kwon SS, Kim J, Kim HW, et al. Efficient strategy for the molecular diagnosis of intractable early-onset epilepsy using targeted gene sequencing. BMC Med Genomics 2018; 11(1):6. doi:10.1186/s12920018-0320-7.

9. Mercimek-Mahmutoglu S, Patel J, Cordeiro D, Hewson S, Callen D, Donner EJ, et al. Diagnostic yield of genetic testing in epileptic encephalopathy in childhood. Epilepsia 2015; 56(5):707-16. doi:10.1111/epi.12954.

10. Parrini E, Marini C, Mei D, Galuppi A, Cellini E, Pucatti D, et al. Diagnostic targeted resequencing in 349 patients with drug-resistant pediatric epilepsies Identifies causative mutations in 30 different genes. Hum Mutat 2017; 38(2):216225. doi:10.1002/humu.23149.

11. Butler KM, da Silva C, Alexander JJ, Hegde M, Escayg A. Diagnostic yield from 339 epilepsy patients screened on a clinical gene panel. Pediatr Neurol 2017; 77:61-66. doi:10.1016/j.pediatrneurol.2017.09.003.

12. Ortega-Moreno L, Giráldez BG, Soto-Insuga V, Losada-Del Pozo R, Rodrigo-Moreno M, Alarcón-Morcillo C, et al. Molecular diagnosis of patients with epilepsy and developmental delay using a customised panel of epilepsy genes. PLoS One 2017; 12(11):e0188978. doi:10.1371/journal.pone.0188978. 
13. Sánchez Fernández I, Loddenkemper T, Gaínza-Lein M, Sheidley BR, Poduri A. Diagnostic yield of genetic tests in epilepsy: A meta-analysis and cost-effectiveness study. Neurol 2019; 92(5):e418-e28. doi:10.1212/WNL.0000000 000006850.

14. Helbig KL, Lauerer RJ, Bahr JC, Souza IA, Myers CT, Uysal B, et al. De Novo pathogenic variants in cacnale cause developmental and epileptic encephalopathy with contractures, macrocephaly, and dyskinesias. Am J Hum Genet 2018; 103(5):666-78. doi:10.1016/j.ajhg.2018.09.006.

15. Česká K, Aulická Š, Horák O, Danhofer P, Říha P, Mareček R, et al. Autosomal dominant temporal lobe epilepsy associated with heterozygous reelin mutation: $3 \mathrm{~T}$ brain MRI study with advanced neuroimaging methods. Epilepsy Behav Case Rep 2018; 11:39-42. doi:10.1016/j.ebcr.2018.10.003.

16. Zhao Q, Liu Z, Hu Y, Fang S, Zheng F, Li X, et al. Different experiences of two PRRT2-associated self-limited familial infantile epilepsy. Acta Neurol Belg 2020; 120(4): 1025-1028. doi:10.1007/s13760-020-01348-9.

17. Liu YD, Ma MY, Hu XB, Yan H, Zhang YK, Yang HX, et al. Brain proteomic profiling in intractable epilepsy caused by tsc1 truncating mutations: A small sample study. Front Neurol 2020; 11:475. doi:10.3389/fneur.2020.00475.

18. Kang JQ, Macdonald RL. Molecular pathogenic basis for
GABRG2 mutations associated with a spectrum of epilepsy syndromes, from generalised absence epilepsy to dravet syndrome. JAMA Neurol 2016; 73(8):1009-16. doi:10.1001/jamaneurol.2016.0449.

19. Wolff M, Brunklaus A, Zuberi SM. Phenotypic spectrum and genetics of SCN2A-related disorders, treatment options, and outcomes in epilepsy and beyond. Epilepsia 2019; 60 Suppl 3:S59-S67. doi:10.1111/epi.14935.

20. Sasai N, Toriyama M, Kondo T. Hedgehog signal and genetic disorders. Front Genet 2019; 10:1103. doi:10.3389/fgene.2019.01103.

21. Pascual O, Traiffort E, Baker DP, Galdes A, Ruat M, Champagnat J. Sonic hedgehog signalling in neurons of adult ventrolateral nucleus tractus solitarius. Eur J Neurosci 2005; 22(2):389-96. doi:10.1111/j.1460-9568.2005.04223.x.

22. Mitchell N, Petralia RS, Currier DG, Wang YX, Kim A, Mattson $M P$, et al. Sonic hedgehog regulates presynaptic terminal size, ultrastructure and function in hippocampal neurons. J Cell Sci 2012; 125(Pt 18):4207-13. doi:10.1242/jcs.105080.

23. Feng S, Ma S, Jia C, Su Y, Yang S, Zhou K, et al. Sonic hedgehog is a regulator of extracellular glutamate levels and epilepsy. EMBO Rep 2016; 17(5):682-94. doi:10.15252/ embr.201541569. 